\title{
Hadron spectrum with domain-wall valence quarks on an improved staggered sea
}

LHPC Collaboration: R.G. Edwards ${ }^{a}$, G. Fleming ${ }^{b}$, Ph. Haegler ${ }^{c}$, C. Morningstar ${ }^{d}$, J.W. Negele ${ }^{e}$, K. Orginos ${ }^{a, f}$, A.V. Pochinsky ${ }^{e}$, D.B. Renner ${ }^{g}$, D.G. Richards ${ }^{*}{ }^{\dagger}$, W. Schroers ${ }^{h}$

a Thomas Jefferson National Accelerator Facility, Newport News, VA 23606, USA

${ }^{b}$ Sloane Physics Laboratory, Yale University, New Haven, CT 06520, USA

${ }^{c}$ Institut für Theoretische Physik, TU München, D-85747 Garching, Germany

${ }^{d}$ Department of Physics, Carnegie-Mellon University, Pittsburgh, PA 15213, USA

${ }^{e}$ Center for Theoretical Physics, Massachusetts Institute of Technology, Cambridge, MA 02139, USA

${ }^{f}$ Department of Physics, College of William and Mary, Williamsburg VA 23187, USA

${ }^{g}$ University of Arizona, Department of Physics, 1118 E 4th St, Tucson AZ 85721, USA

${ }^{h}$ John von Neumann-Institut für Computing NIC/DESY, D-15738 Zeuthen, Germany

The hadron spectrum is computed in full QCD using domain-wall valence fermions on an improved staggered sea, for pion masses down to around $350 \mathrm{MeV}$. Emphasis is laid on the lowlying baryon spectrum. All possible baryon correlators obtainable from local and quasi-local quark sources are computed, using lattice group-theory methods. Results are presented for the lowest-lying states in each isospin channel.

XXIVth International Symposium on Lattice Field Theory

July 23-28, 2006

Tucson, Arizona, USA

\footnotetext{
* Speaker.

†Email: dgr@lab.org
} 


\section{Introduction}

The calculation of the spectrum of QCD using the lattice is a benchmark test of the lattice method. The lowest-lying states are well-determined through experiment, whilst the spectrum of excited resonances provides insight into the effective degrees of freedom of $\mathrm{QCD}$, both to guide experiment and to verify the range of validity of QCD-inspired models of hadrons, and is thus the focus of increasing effort[1, 2, 3, 4].

The LHP Collaboration has engaged in an extensive program to study hadron structure, using a domain-wall fermion formulation for the valence quarks, computed on an ensemble of configurations generated by the MILC collaboration using Asqtad fermions for the sea quarks. The MILC collaboration has performed an extensive investigation of the spectrum using Asqtad valence quarks; thus the study initiated in this talk will provide an important investigation of the systematics of the hybrid formulation. The construction of baryon interpolating operators using staggered quarks is delicate, complicated by taste-symmetry breaking. Thus a investigation of the baryon spectrum using DWF valence propagators is particularly valuable, and is the focus of this talk.

\section{Interpolating Operators}

The cubic lattice employed in our calculations does not admit the full rotational symmetry of the continuum, but rather the more restricted symmetry of the octahedral group. Thus states at rest are classified according to the irreducible representation (irreps) of the cubic group, and for spectroscopy calculations, interpolating operators must be constructed that transform irreducibly under the lattice group. A procedure for constructing such operators was described in two papers, the first of which employed a Clebsch-Gordon approach mirroring that of the continuum [5], and the second an automated method allowing a very general basis of operators to be constructed [6].

A comprehensive study of the baryon resonance spectrum, in which many energy eigenvalues are extracted in each quantum channel, requires the computation of many quark propagators at each quark mass, and is therefore computationally highly demanding, even in the quenched approximation to QCD. In this talk, we consider only those operators that can be constructed from local or quasi-local quark sources and sinks, and in fact the propagators employed in our studies of hadron structure. The basic building blocks are the three-quark interpolating operators of the form:

$$
\bar{B}(x)=\tilde{\bar{\psi}}_{\alpha}^{A, i} \tilde{\bar{\psi}}_{\beta}^{B, j} \tilde{\bar{\psi}}_{\gamma}^{C, k} \varepsilon^{i j k}
$$

where $A, B, C$ denote flavor $(u / d$ or $s), i, j, k$ denote color, and $\alpha, \beta, \gamma$ are Dirac indices. The tilde signifies that the fields may be Gaussian smeared.

There are three double-valued irreducible representations of the cubic group, denoted $G_{1 u / g}$, $H_{u / g}$ and $G_{2 u / g}$, where $g$ and $u$ refer to positive and negative parity, respectively; $G_{1}$ contains continuum spins $1 / 2,7 / 2, \ldots, H_{g}$ spins $3 / 2,5 / 2, \ldots$ and $G_{2}$ spins $5 / 2,7 / 2, \ldots$. The number of linearly independent interpolating operators that can be formed from $u / d$ and $s$ quark quasi-local fields, classified according to the irreducible representations of the cubic group, is shown in Table 1. Only irreps $G_{1 g / u}$ and $H_{g / u}$ are accessible with these propagators, and thus spins 5/2 and greater occur only as sub-leading states in these irreps. However, the assumption, implicit in many lattice calculations, that the lightest state in any irrep is that having the lowest spin may well be erroneous; 


\begin{tabular}{cccc} 
Flavor & $G_{1 g / u}(2)$ & $H_{g / u}(4)$ & $G_{2 g / u}(2)$ \\
\hline$N$ & 3 & 1 & - \\
$\Delta$ & 1 & 2 & - \\
$\Lambda$ & 4 & 1 & - \\
$\Sigma$ & 4 & 3 & - \\
$\Xi$ & 4 & 3 & - \\
$\Omega$ & 1 & 2 & -
\end{tabular}

Table 1: The number of linearly independent interpolating operators that can be formed from $u / d$ and $s$ quark quasi-local fields in each irreducible representation of the cubic group. The number in brackets denotes the dimension of the representation.

\begin{tabular}{ccc}
$m_{u / d}$ & $m_{s}$ & Number "Configs" \\
\hline 0.04 & 0.05 & 171 \\
0.03 & 0.05 & 700 \\
0.02 & 0.05 & 1455 \\
0.01 & 0.05 & 1305 \\
\hline
\end{tabular}

Table 2: The number of propagators at each value of the light $(u / d)$ and strange $(s)$ quark masses; each propagator is computed on up to the four possible bisections of the $20^{3} \times 64$ gauge configurations, as discussed in the text.

in the nucleon sector, lattice calculations suggest that the lightest spin-5/2 state lies at or below that of spin-3/2 [7]. Focusing on the familiar $I=1 / 2$ nucleon operators, we see that there are three $G_{1}$ operators of each parity, and a single $H$ operator of each parity; these correspond to the two familiar Ioffe currents used as an interpolating field, together with the spin- $1 / 2$ and spin- $3 / 2$ components in the decomposition of the Rarita-Schwinger construction.

\section{Computational Details}

We employ the $20^{3} \times 64$ gauge configurations at lattice spacing $a \simeq 0.12 \mathrm{fm}$ generated by the MILC Collaboration, using an Asqtad formulation for the sea quarks [8]. We use the lattice spacing $a=0.124 \mathrm{fm}$ determined from heavy-quark spectroscopy[9]. Valence quark propagators corresponding to both the $u / d$ quarks and the $s$ quark are computed on $20^{3} \times 32$ lattices, using a domain-wall fermion action, with the gauge fields first smeared using the HYP procedure and then bisected in the temporal direction. Dirichlet boundary conditions are used in the temporal direction, with the source placed ten time slices from the boundary; we employ Gaussian-smeared sources, and propagators are then computed to both local and Gaussian-smeared sinks. The valence quark masses are determined by tuning the valence pseudoscalar mass to that of the corresponding Asqtad Goldstone boson. For each gauge configuration, propagators are computed from up to four different source points, corresponding to the four possible temporal bisections of the lattice. The parameters of the calculation are listed in Table 2. 


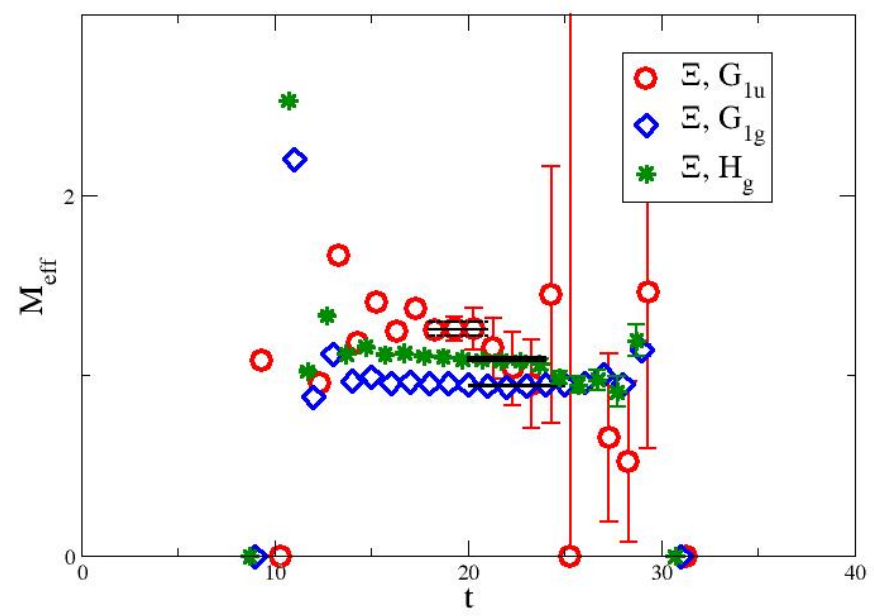

Figure 1: The effective masses in the cascade channel obtained from the smeared-smeared correlators at light-quark masses $m_{u / d}^{\text {asqtad }}=0.03$. The different plotting symbols correspond to the different irreps., $G_{1 g}$, $H_{g}$ and $G_{1 u}$; symbols are displaced for clarity.

\section{Results}

The domain-wall fermion action, whilst local in five dimensions, extends over several temporal time slices in four dimensions. Thus there is no transfer matrix propagating between succeeding time slices. This non-locality is at the scale of the cut-off, and is exhibited particularly clearly in the correlators corresponding to the heavier states. This is illustrated in Figure 1, where we show the effective masses for the cascades in the $G_{1 g}, H_{g}$ and $G_{1 u}$ channels for smeared-smeared correlators, where the residues in the spectral sum are positive definite. Similar behavior is observed in a recent quenched study of charmonium[10], suggesting that this is a manifestation of the non-locality of the action, rather than of the partially quenched nature of the calculation, and correspondingly that computations at finer values of the lattice spacing should progressively alleviate this issue.

The use of a complete matrix of correlators for the smeared-smeared data in principle enables the variational method to be used to extract not only the lowest energy eigenvalue, but also some of the excited eigenvalues, by solving the generalized eigenvalue equation

$$
C(t) u=\lambda\left(t, t_{0}\right) C\left(t_{0}\right) u
$$

to obtain a set of real (ordered) eigenvalues $\lambda_{n}\left(t, t_{0}\right)$, where $\lambda_{0}>\lambda_{1}>, \ldots$. At large Euclidean times, these eigenvalues then delineate between the different masses

$$
\lambda_{n}\left(t, t_{0}\right) \longrightarrow e^{-M_{n}\left(t-t_{0}\right)}+O\left(e^{-M_{n+1}\left(t-t_{0}\right)}\right) .
$$

In practice, though we find that the lowest eigenvalue is indeed well determined, the second and higher eigenvalues are rather poorly determined. Furthermore, the effect of the non-locality in the 


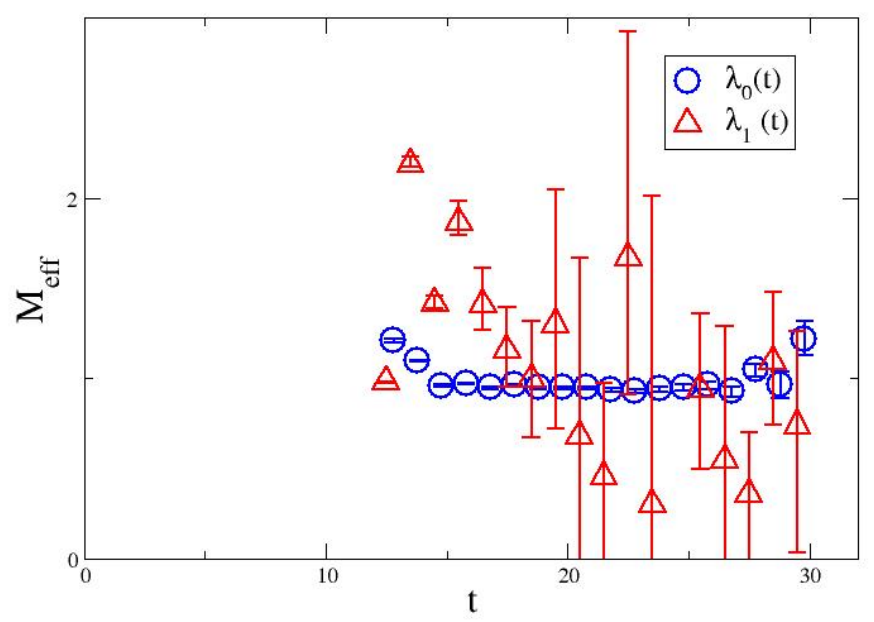

Figure 2: The effective masses obtained from the largest two principle eigenvalues in the solution of the generalized eigenvalue equation, Eqn. 4 , for the $G_{1 / 2}$ cascade channel at quark mass $m=0.03$; symbols are displaced for clarity.

larger eigenvalues are clear, as shown in Figure 2; a more comprehensive investigation of this is in progress.

The ability to extract more than a single principal eigenvalue has been clearly demonstrated in [7], where a broad basis of interpolating operators is employed that probes the spatial structures of the states. In this talk, we present results only for the lowest-lying states in each channel, using single-particle fits to the smeared-local correlators, as those have the lesser statistical noise.

Our preliminary results for the spectrum are summarized in Figure 3; our final results will aim to perform multi-particle fits to the matrix of correlators. Fits to the $\Delta$ resonance at the lighter values of the pion mass are problematical, with no unambiguous plateaux, though it should be noted that the pion masses in this calculation are still sufficiently large for the pion to be stable on these lattices. In general, the quality of the data is insufficient to admit reliable fits to the negative-parity states.

Figure 3 shows the approach to $S U(3)$ symmetry as the $u / d$ quark masses approaches that of the $s$ quark. The investigation of the chiral behavior of both the nucleon and excited resonances is currently a topic under debate. We will not present detailed chiral extrapolations in this talk, but rather show a benchmark quantity for which the leading non-analytic behavior is suppressed. The left-hand panel in Figure 4 shows the mass combination $3 M_{N}-M_{\Xi}$; we see essentially flat behavior with the pion mass, and see good agreement with the physical value. The right-hand panel shows the extent to which the Gellman-Okubo mass relation is satisfied; this was investigated in detail on the same lattices, but using different interpolating operators, or rather a linear combination of our operators, in Ref [11]. 

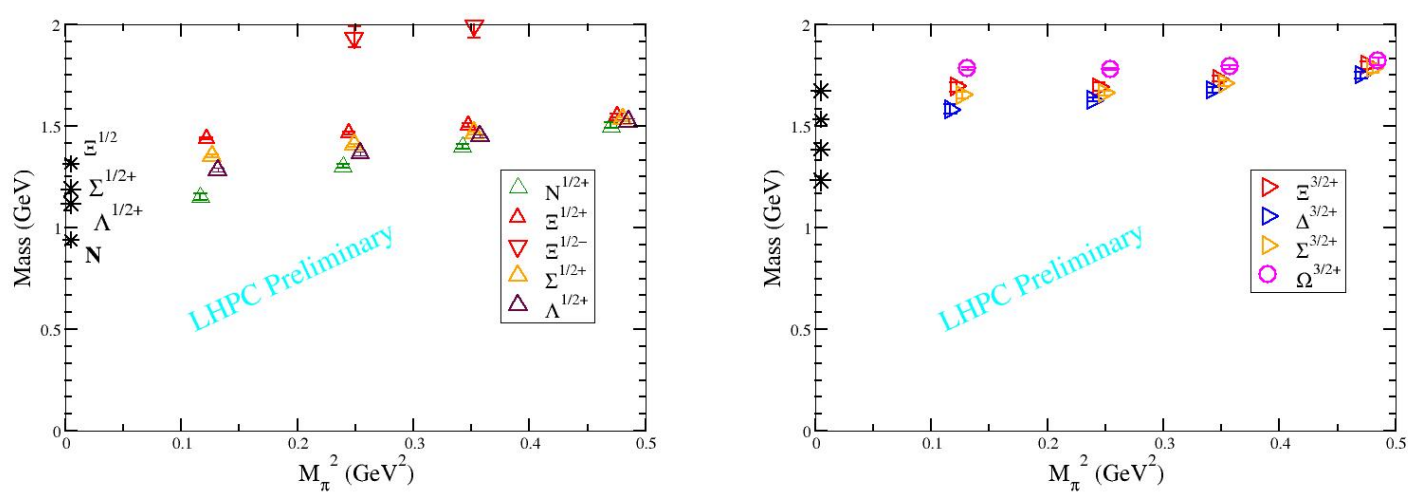

Figure 3: The left-hand figure shows the masses of the low-lying spin- $1 / 2$ states, obtained from singleparticle fits to the smeared-local correlators. The right-hand figure is the plot for spin- $3 / 2$ states, though the difficulty in obtaining reliable fits to the $\Delta$ resonance should be noted. In both figures, plotting symbols are displaced for clarity.
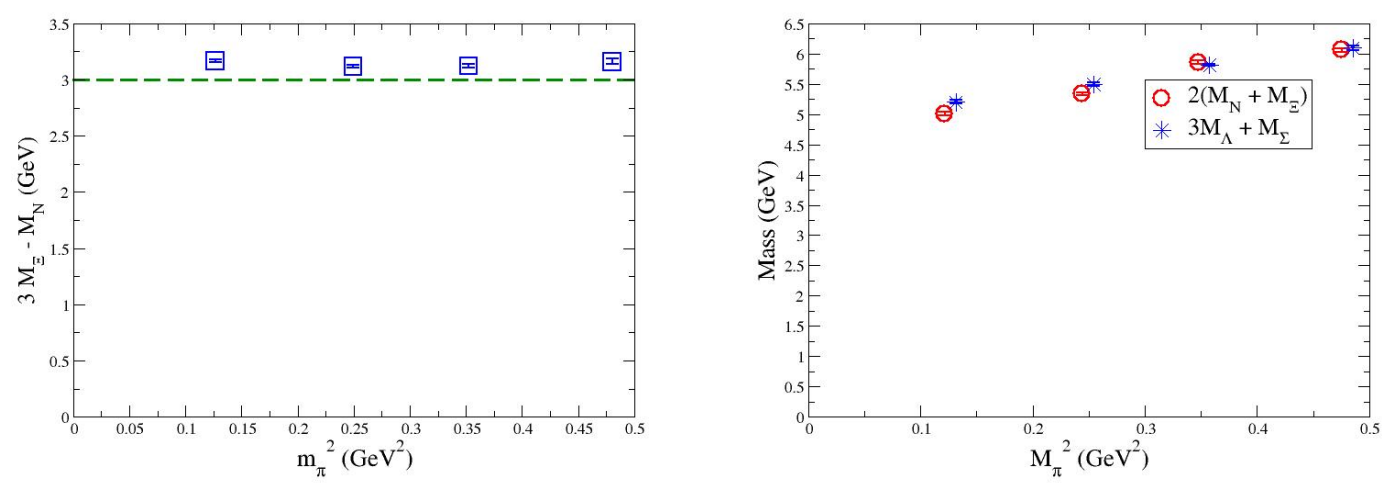

Figure 4: The left-hand panel shows the mass combination $3 M_{N}-M_{\Xi}$, for which the leading chiral behavior cancels, together with the corresponding physical value. The right-hand panel shows the degree to which the Gellman-Okubo mass relations holds in this calculation; symbols are displaced for clarity.

\section{Conclusions}

We have applied the group theoretical methods to measure all the baryon correlators that can constructed with local- or quasi-local propagators alone. Single-particle fits to the correlators enable the preliminary extraction of the ground-state masses for most of the positive-parity spin- $1 / 2$ and spin-3/2 states of each isospin; a more detailed program of fits to the data is in progress. Future work will center on a comparison with the computations using the asqtad fermion action for the valence quarks, and on a study of the chiral extrapolations of the data to the physical values of the pion mass. 


\section{Acknowledgments}

This work was supported by the DOE Office of Nuclear Physics under contracts DE-FC0294ER40818, DE-FG02-92ER40676, and DE-AC05-84ER40150, the EU Integrated Infrastructure Initiative Hadron Physics (I3HP) under contract RII3-CT-2004-506078 and by the DFG under contract FOR 465 (Forschergruppe Gitter-Hadronen-Phänomenologie). Computations were performed on clusters at Jefferson Laboratory and at ORNL using time awarded under the SciDAC initiative. We are grateful to the MILC collaboration for the use of the gauge configurations used in this study, and to the NPLQCD Collaboration for the use of the strange-quark propagators.

\section{References}

[1] N. Mathur et al., Phys. Lett. B605 (2005) 137, hep-ph/0306199.

[2] CSSM Lattice, J.M. Zanotti et al., Phys. Rev. D68 (2003) 054506, hep-lat/0304001.

[3] K. Sasaki and S. Sasaki, Phys. Rev. D72 (2005) 034502, hep-lat/0503026.

[4] T. Burch et al., Phys. Rev. D74 (2006) 014504, hep-lat/0604019.

[5] Lattice Hadron Physics (LHPC), S. Basak et al., Phys. Rev. D72 (2005) 074501, hep-lat/0508018.

[6] LHP, S. Basak et al., Nucl. Phys. Proc. Suppl. 140 (2005) 287, hep-lat/0409093.

[7] S. Basak et al., (2006), hep-lat/0609052.

[8] C.W. Bernard et al., Phys. Rev. D64 (2001) 054506, hep-lat/0104002.

[9] C. Aubin et al., Phys. Rev. D70 (2004) 094505, hep-lat/0402030.

[10] J.J. Dudek, R.G. Edwards and D.G. Richards, Phys. Rev. D73 (2006) 074507, hep-ph/0601137.

[11] S.R. Beane, K. Orginos and M.J. Savage, (2006), hep-lat/0604013. 